\title{
The optimal monoenergetic spectral image level of coronary computed tomography (CT) angiography on a dual-layer spectral detector CT with half-dose contrast media
}

\author{
Xin Huang ${ }^{1}$, Sizhe Gao ${ }^{1}$, Yue $\mathrm{Ma}^{1}$, Xiaomei Lu ${ }^{2}$, Zheng Jia ${ }^{2}$, Yang Hou ${ }^{1}$ \\ ${ }^{1}$ Department of Radiology, Shengjing Hospital of China Medical University, Shenyang 110004, China; ${ }^{2}$ CT Clinical Science, Philips Healthcare, \\ Shenyang 110016, China
}

Correspondence to: Yang Hou. Department of Radiology, Shengjing Hospital of China Medical University, No. 36, Sanhao Street, Heping District, Shenyang 110004, China. Email: houyang1973@126.com.

Background: To investigate the optimal monoenergetic level of spectral reconstructions in coronary computed tomography angiography (coronary CTA) on a dual-layer spectral detector computed tomography (SDCT) with half-dose contrast media.

Methods: Two hundred patients with suspected coronary artery disease (CAD) were enrolled in this prospective coronary CTA study and randomly divided into a routine-dose contrast media group and a half-dose contrast media group (each $\mathrm{n}=100$ ). Coronary CTA was performed using SDCT with prospective electrocardiogram (ECG)-gated mode. A tube voltage of $120 \mathrm{kVp}$ was used, along with an automated tube current modulation. A dose of iodixanol $270 \mathrm{mgI} / \mathrm{mL}$ of 0.8 and $0.4 \mathrm{~mL} / \mathrm{kg}$ was administered to the routine and half-dose groups, respectively. For the routine-dose group, $120 \mathrm{kVp}$ polychromatic images with a model-based iterative reconstruction (IMR) (Group A) were reconstructed. For the half-dose group, three monoenergetic levels of images were reconstructed (Group B, $45 \mathrm{keV}$; Group C, $50 \mathrm{keV}$; and Group D, $55 \mathrm{keV}$ ). Objective indicators [mean CT values; noise; signal-to-noise ratio (SNR); and contrast-to-noise ratio (CNR)] and subjective indicators (contrast, sharpness, subjective noise, and acceptability) in each group were compared.

Results: There were no significant differences in demographics or radiation dose $(1.83 \pm 0.51$ vs. $1.80 \pm$ $0.53 \mathrm{mSv}, \mathrm{P}=0.78$ ) between the routine- and half-dose groups. The average iodine loads were $15.33 \pm 2.26$ and 7.48 \pm 1.14 g, respectively. Mean CT values, SNR, CNR, and subjective contrast in Group C were higher than those in Group A $(\mathrm{P}<0.05)$, and there were no significant differences in other indicators between Group $\mathrm{C}$ and Group A $(\mathrm{P}>0.05)$. The objective and subjective noise in Group B were worse than those in Group A $(\mathrm{P}<0.05)$. The contrast, sharpness, and acceptability of Group D were all worse than those of Group A $(\mathrm{P}<0.05)$.

Conclusions: Compared to routine polychromatic images, $50 \mathrm{keV}$ monoenergetic images can provide equivalent or improved coronary image quality in coronary CTA performed on SDCT with half the amount of contrast media.

Keywords: Contrast media; coronary computed tomography angiography (coronary CTA); dual-layer spectral detector computed tomography (dual-layer SDCT); image quality; virtual monoenergetic image (VMI)

Submitted Jul 10, 2019. Accepted for publication Feb 18, 2020.

doi: $10.21037 /$ qims.2020.02.17

View this article at: http://dx.doi.org/10.21037/qims.2020.02.17 


\section{Introduction}

Due to its high sensitivity, coronary computed tomography angiography (coronary CTA) has become the preferred non-invasive modality for the chest pain work-up of patients with low to intermediate risk of coronary artery disease (CAD) (1-4). However, the increased awareness of the use of contrast media in CT in general, has raised concerns of contrast media-induced nephropathy $(\mathrm{CIN})(5,6)$. In recent years, there has been an intense debate on the cause-and-effect of iodinated contrast media and nephrotoxicity $(7,8)$. Nevertheless, data are indicating a potential nephrotoxic effect from intravenous contrast media $(9,10)$. Nyman et al. (11) found that the incidence of acute renal injury induced by contrast media was closely related to the volume load and concentration of iodine. At a contrast media-dose/estimated glomerular filtration rate (eGFR) ratio $<1$, the risk of CIN was $3 \%$, increasing to $25 \%$ when the ratio was $\geq 1$. The Society of Cardiovascular Computed Tomography (SCCT) guidelines for the performance and acquisition of coronary CTA recommend the minimization of contrast media volume (12). Therefore, it is of great importance that a load of iodinated contrast media is reduced while simultaneously maintaining the diagnostic image quality in coronary CTA. One of the common approaches to contrast media load reduction is to lower the tube energy (voltage). The use of low tube voltage $(70 / 80 / 100 \mathrm{kVp})$ brings the peak $\mathrm{X}$-ray energy closer to the k-edge of iodine $(33 \mathrm{keV})$, resulting in a larger photoelectric effect. This increases the degree of vascular attenuation, which could enable a reduction in the volume of contrast media in a range of $12-56 \%$ in comparison with the use of higher tube voltages $(13,14)$. However, the use of low tube voltage is limited to patients with small/moderate body habitus or body mass index (BMI) (e.g., BMI $\leq 25 \mathrm{~kg} / \mathrm{m}^{2}$ ) (15). Such an approach is also accompanied by an increase of image noise, which can sometimes hamper diagnosis, especially in areas of complex anatomy and small vessels (16).

Similarly, the use of virtual monoenergetic images (VMIs) of dual-energy CT (DECT) is another way to effectively reduce contrast media dose, with several studies demonstrating the use of low contrast volumes facilitated by DECT in coronary, pulmonary, aortic, abdominal, and lower-extremity CTA (17-24). Other DECT investigations using rapid $\mathrm{kVp}$ switching showed that the mean iodine administered can be reduced to $11.2 \mathrm{~g}$ (calculated from the absolute volume of contrast $35 \mathrm{~mL}$ and contrast media concentration $320 \mathrm{mgI} / \mathrm{mL}$ ) and $12.5 \mathrm{~g}$ at $60 \mathrm{keV}$ in coronary CTA $(18,19)$. The reason for not further reducing contrast load was that the image noise increases significantly at lower VMIs.

The dual-layer spectral detector CT (SDCT) enables simultaneous acquisition of low- and high-energy projection data and generates a variety of spectral reconstructions. The top layer absorbs low-energy photons, with the bottom layer absorbing high-energy photons, thereby simultaneously generating projection data from the two detector layers corresponding to high and low energy at the same spatial and angular location. One of the benefits of the detector-based approach is that the projection data from the two detector layers are in near-perfect alignment, thus taking advantage of the anti-correlated nature of the noise in the photoelectric and Compton scatter images by canceling them out (25-27). This results in reduced noise levels even at energy levels $<60 \mathrm{keV}$, thus making it possible to achieve further contrast enhancement and improvement of signal and SNR without impairing image quality.

van Hamersvelt et al. (28) found that it was feasible to reduce iodine concentration by $60 \%$ in the circulation phantom when using SDCT at $40 \mathrm{keV}$ without loss of objective image quality compared to routine iodine concentration images at $120 \mathrm{kVp}$. Yi et al. (29) experimented with $36 \mathrm{~mL}(370 \mathrm{mgI} / \mathrm{mL})$ of contrast media volume in 60 patients ( 30 patients at $120 \mathrm{kVp}$ and 30 patients at $100 \mathrm{kVp}$ ). Their study results showed that $40-50 \mathrm{keV}$ monoenergetic spectral reconstructions from $120 \mathrm{kVp}$ coronary CTA on SDCT provide improved coronary image quality compared to conventional reconstructions obtained from $100 \mathrm{kVp}$ scans. To the best of our knowledge, there are no in vivo reports with large samples and individualized application of low dose contrast media that draw a comparison between the image quality of monoenergetic images and polychromatic images in coronary CTA. Therefore, in this study, we discuss the feasibility of reducing contrast media by $50 \%$ and the best low $\mathrm{keV}$ monoenergetic spectral coronary CTA in the application of SDCT by comparing polychromatic images with the routine contrast media.

\section{Methods}

\section{Study population}

This single-center, randomized, prospective study was approved and supervised by the Institutional Committee of Ethics of Shengjing Hospital of China Medical University. All patients gave informed consent.

This study included clinically suspected CAD patients 
referred to coronary CTA due to chest pain, angina pectoris, or pending stress tests between November 2017 and February 2018. Exclusion criteria included: known allergies to iodinated contrast media, renal insufficiency (eGFR $<60 \mathrm{~mL} /$ minute $/ 1.73 \mathrm{~m}^{2}$ or serum creatinine level $\geq 120 \mathrm{mmol} / \mathrm{L}$ ), pregnancy, severe arrhythmia, cardiac function less than grade III, and patients with coronary stents or coronary artery bypass grafts. A total of 200 eligible patients participated in this coronary CTA study. Each patient was assigned at random to either the routine iodine load polychromatic coronary CTA group $(n=100)$ or the half iodine load monoenergetic coronary CTA group $(n=100)$ by way of a random number method.

\section{Coronary CTA scan protocols}

All patients underwent coronary CTA examinations on a dual-layer SDCT (IQon, Philips Healthcare, Best, The Netherlands) using prospective electrocardiogram (ECG)gated acquisitions (Step \& Shoot Cardiac). The automatic bolus tracker was used with a region of interest (ROI) in the ascending aorta at the level of the pulmonary artery. The scans were initiated under full inspiration $6 \mathrm{~s}$ after a predetermined signal attenuation threshold of $150 \mathrm{HU}$ (routine iodine load group) and $90 \mathrm{HU}$ (half iodine load group) were attained. Contrast media (Visipaque Iodixanol 270; GE Healthcare, Ireland) was injected intravenously through the antecubital vein using an 18-gauge catheter dual-tube highpressure syringe (Ulrich REF XD 2051). Contrast media was used on an individual basis according to body weight. In the routine iodine load group, the total amount of contrast media $=$ patient weight $\times 0.8 \mathrm{~mL} / \mathrm{kg}$. The half iodine load group was treated with a mixture of contrast media and normal saline (NS) (contrast media: NS $=1: 1$ ), and the total amount of mixture $=$ patient weight $\times 0.8 \mathrm{~mL} / \mathrm{kg}$. The same injection flow rate was used for both groups. Contrast injection flow rate $(\mathrm{mL} / \mathrm{s})=$ total amount $(\mathrm{mL}) /$ injection time (12 s) (30), followed by injection of $30 \mathrm{~mL}$ of saline at the same injection rate. For patients with a weight exceeding $90 \mathrm{~kg}$, the highest injection rate was $6 \mathrm{~mL} / \mathrm{s}$. The scan parameters were as follows: tube voltage was set to $120 \mathrm{kVp}$; tube current automatic exposure control [Dose Right Index (DRI) =13] was used; field of view $=250 \mathrm{~mm}$; tube rotation time $=0.27 \mathrm{~s}$; detector collimation $=64 \times 0.625 \mathrm{~mm}$; slice thickness $=0.9 \mathrm{~mm}$; increment $=0.45 \mathrm{~mm}$; and matrix $=512 \times 512$. The scan trigger was centered around a physiologic cardiac phase of ventricular diastasis corresponding to $78 \%$ of the R-R interval with a $\pm 3 \%$ buffer. Before CT examination, patients with a heart rate $(\mathrm{HR})>75 \mathrm{bpm}$ received a $\beta$-receptor blocker 25-50 mg (Metoprolol Succinate sustained-release tablets, AstraZeneca, Sweden) orally to reduce and stabilize HR.

\section{Image reconstruction}

Images in the routine iodine load group (Group A) were reconstructed using model-based iterative reconstruction (IMR Cardiac Routine Level 1, Philips Healthcare). Images in the half iodine load group were reconstructed using the vendor-recommended spectral reconstruction level (Spectral Recon Level 4, Philips Healthcare) with these datasets being spectral based images (SBI). Three monoenergetic image series—45 keV (Group B), $50 \mathrm{keV}$ (Group C), and $55 \mathrm{keV}$ (Group D)-were used in this study. All reconstructions were targeted to provide a spatial resolution of the cardiac standard (CB) reconstruction kernel.

\section{Qualitative analysis}

Data was transferred to the dedicated CT workstation (IntelliSpace Portal Version 6.5, Philips Healthcare), with advanced cardiac application software (Cardiac Viewer and Comprehensive Cardiac Analysis) used to review the image data of each group. The optimal coronary artery phase (minimum motion artifact image) was selected for image evaluation. Transverse images, multi-planar reformation, and a volume rendering technique were used for the data assessment. The subjective image quality of three coronary arteries [left anterior descending (LAD), left circumflex (LCX), and right coronary artery (RCA)] was evaluated for contrast between blood vessels and surrounding tissues, lumen edge sharpness, subjective noise, and overall image acceptability in vessels with a diameter $\geq 1.5 \mathrm{~mm}$ (4). Two experienced radiologists (with $\geq 5$ years in cardiovascular CT and diagnostic experience) independently performed a 5-point Likert scoring system (1 and 2: poor contrast, very blurred lumen edge and high noise, impossible to diagnose; 3: suboptimal contrast, blurred lumen edge and high noise, sufficient to exclude obstructive disease; 4: good contrast, sharp lumen edge, and low noise, preserved ability to evaluate the degree of stenosis and identify mild atherosclerosis; and 5: excellent contrast, very clear and sharp lumen edge, and very low noise; ability to simultaneously evaluate the existence of obstructive disease and mild atherosclerosis) $(18,19)$. The coronary CTA images were viewed with the initial window width 
Table 1 Patient characteristics

\begin{tabular}{lcccc}
\hline Item & $\begin{array}{c}\text { Group A } \\
(\mathrm{n}=100)\end{array}$ & $\begin{array}{c}\text { Group B-D } \\
(\mathrm{n}=100)\end{array}$ & $\mathrm{F}$ & P value \\
\hline Age (year) & $55.72 \pm 10.17$ & $55.26 \pm 10.94$ & 0.049 & 0.758 \\
$\begin{array}{l}\text { Gender (male/ } \\
\text { female) }\end{array}$ & $60 / 40$ & $56 / 44$ & 1.234 & 0.569 \\
Height $(\mathrm{cm})$ & $167.62 \pm 7.52$ & $168.94 \pm 7.27$ & 1.382 & 0.209 \\
Weight $(\mathrm{kg})$ & $70.94 \pm 11.09$ & $69.60 \pm 11.24$ & 0.114 & 0.397 \\
BMl $\left(\mathrm{kg} / \mathrm{m}^{2}\right)$ & $25.18 \pm 3.02$ & $24.36 \pm 3.38$ & 0.018 & 0.073 \\
Heart rate & $59.44 \pm 5.11$ & $60.22 \pm 4.94$ & 0.280 & 0.274 \\
Heart rate & $3.62 \pm 3.23$ & $3.42 \pm 2.61$ & 1.002 & 0.630 \\
variability & & & & \\
\hline
\end{tabular}

Data are presented as the mean \pm standard deviation or number. BMI, body mass index.

and window level set to 750 and $90 \mathrm{HU}$, respectively. The readers can adjust the precise window width and window level for the qualitative assessment of image quality. The readers were blinded to the reconstruction protocol and scanning conditions. If necessary, the opinion of a third radiologist was used to reconcile differences to obtain a consensus.

\section{Quantitative analysis}

Objective evaluation indicators include image signal intensity (CT value), image noise (standard deviation, $\mathrm{SD}$ ), signal-to-noise ratio (SNR), and contrast-to-noise ratio (CNR). The ROI was placed in the aortic root at the origin of the left main coronary artery, and distal segments of the LAD, LCX, and RCA, and adjacent perivascular fatty tissue. All ROIs were selected in the homogeneous areas to avoid the edges of the vessel or surrounding tissues. The sizes of ROI were $1.5 \mathrm{~cm}^{2}$ in the aortic root and were adapted to 0.2 to $0.4 \mathrm{~cm}^{2}$ in the coronary arteries. The mean $\mathrm{CT}$ values (HU) in the ROIs were measured, and the SD of the ROI in the aortic root was taken as the image noise. The SNR and CNR were calculated as follows: $\mathrm{SNR}=$ signal $_{\text {arteries }}$ ' noise $_{\text {ao }} ; \mathrm{CNR}=\left(\mathrm{HU}_{\text {arteries }}-\mathrm{HU}_{\mathrm{fat}}\right) /$ image noise $_{\mathrm{ao}}(31)$. For each patient, the shape, position, and size of the ROI were kept consistent for all measurements.

\section{Coronary CTA radiation dose measurement}

The effective radiation dose for each patient was determined using the CT dose index $\left(\mathrm{CTDI}_{v o l}\right)$ and the dose length product (DLP). The effective dose (ED) was obtained as the product of the DLP and a conversion coefficient for chest $\mathrm{k}$ ( $\mathrm{k}=0.014 \mathrm{mSv} / \mathrm{mGy} / \mathrm{cm})(32)$.

\section{Statistical analysis}

Statistical analyses were performed using the SPSS Statistics software (version 23.0, IBM). Continuous variables are expressed as mean $\pm \mathrm{SD}$. The independent samples $t$-test was used to compare demographic data [age, gender, HR, heart rate variability (HRV), height, weight, BMI], and radiation dose $\left(\mathrm{CTDI}_{\text {vol }}\right.$, DLP, ED). One-way ANOVA analysis or Welch's $t$-test was used to compare objective evaluation indices (mean CT values, SD, SNR, and CNR) and the least significant difference (LSD) method or Dunnett's T3 test was used for pairwise comparison. A Kappa test was used for the inter-reader agreement of subjective evaluation of image quality. Subjective evaluation indicators (contrast, sharpness, subjective noise, and acceptability) of image quality between groups were compared using the Mann-Whitney $U$ test. A value of $\mathrm{P}<0.05$ was considered statistically significant.

\section{Results}

All 200 patients (116 men and 84 women) completed their coronary CTA exams successfully. The demographic characteristics did not differ between the groups $(\mathrm{P}>0.05)$ (Table 1).

\section{Comparison of subjective evaluation indexes of image quality}

The subjective ratings of the two radiologists were very consistent among the groups. The Kappa values for image contrast, sharpness, subjective noise, and acceptability were $0.80,0.86,0.83$, and 0.85 , respectively. There were no significant differences in sharpness, subjective noise, and acceptability between Group $\mathrm{C}$ and Group A ( $\mathrm{P}=0.368,0.091$, and 0.800, respectively), and the contrast in Group C was superior to that of Group A $(\mathrm{P}<0.001)$. The contrast in Group B was higher than that in Group $\mathrm{A}(\mathrm{P}<0.001)$, but the subjective noise was worse than that of Group A $(\mathrm{P}<0.001)$. The contrast, sharpness, and acceptability of Group D were all worse than those of Group A $(\mathrm{P}<0.001)$ (Table 2; Figure 1). Figure 2 shows representative cases. 
Table 2 Comparison of subjective observation indices of image quality among all groups

\begin{tabular}{|c|c|c|c|c|c|c|c|}
\hline Item & $\begin{array}{c}\text { Group A } \\
(1 / 2 / 3 / 4 / 5)^{\star}\end{array}$ & $\begin{array}{c}\text { Group B } \\
(1 / 2 / 3 / 4 / 5)^{\star}\end{array}$ & $\begin{array}{c}\text { Group C } \\
(1 / 2 / 3 / 4 / 5)^{\star}\end{array}$ & $\begin{array}{c}\text { Group D } \\
(1 / 2 / 3 / 4 / 5)^{\star}\end{array}$ & $\mathrm{P}$ value (A vs. B) & $P$ value (A vs. C) & $P$ value (A vs. D) \\
\hline Contrast & 0/0/14/30/56 & 0/0/0/2/98 & 0/0/0/16/84 & $0 / 0 / 20 / 56 / 24$ & $<0.001$ & $<0.001$ & $<0.001$ \\
\hline Sharpness & $0 / 0 / 6 / 32 / 62$ & 0/0/8/34/58 & $0 / 0 / 8 / 36 / 56$ & $0 / 0 / 16 / 58 / 26$ & 0.523 & 0.368 & $<0.001$ \\
\hline Subjective noise & 0/0/2/32/66 & 0/0/20/42/38 & 0/0/12/36/52 & 0/0/12/24/64 & $<0.001$ & 0.091 & 0.435 \\
\hline
\end{tabular}

*, $(1 / 2 / 3 / 4 / 5)$ represents the scores of each subjective evaluation index.
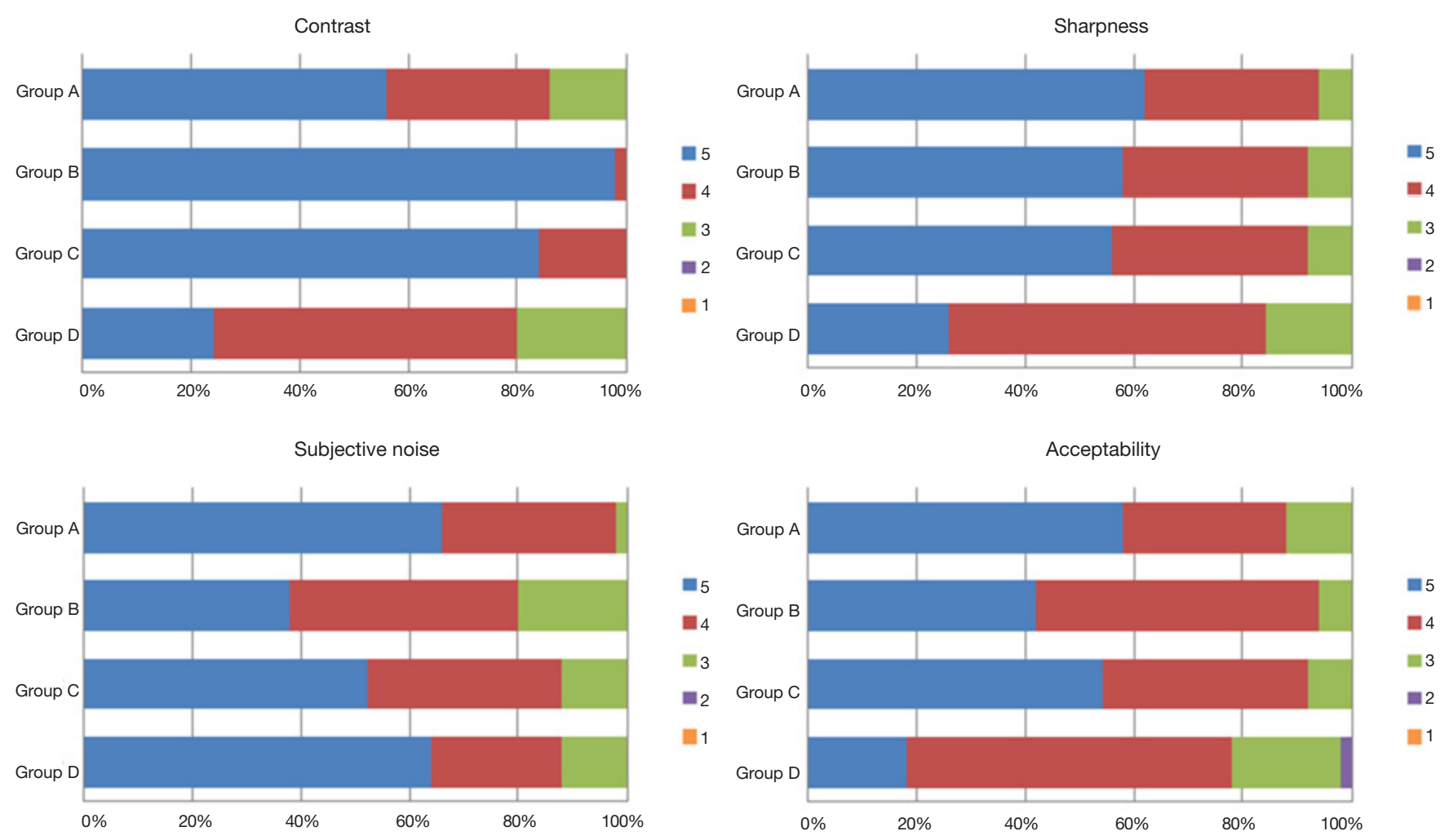

Figure 1 Subjective image quality score for routine-dose Group A (120 kVp) and half-dose Group B-D (45/50/55 keV). The monoenergetic $50 \mathrm{keV}$ spectral images of Group C showed similar subjective image quality scores in sharpness, subjective noise, and acceptability to the conventional $120 \mathrm{kV}$ p polychromatic images of Group A, and had significantly more images that were scored 5 in contrast.

\section{Comparison of objective evaluation indexes of image quality}

Significant differences in objective evaluation indexes were observed among the groups (all $\mathrm{P}<0.05$ ). The CT value, $\mathrm{SNR}$, and CNR in Group $\mathrm{C}$ were higher than those in Group A in all evaluated locations, and there was no significant difference in SD between Group C and Group A $(\mathrm{P}=0.75)$. In all evaluated locations, the $\mathrm{CT}$ value, $\mathrm{SNR}$, and CNR in Group B were higher than those in Group A, but the SD in Group B was also larger than that in Group $\mathrm{A}(\mathrm{P}<0.05)$. There was no significant difference between the objective scores of Group D and Group A in all evaluated locations (Table 3; Figure 3).

\section{Contrast media load and radiation dose}

There was no significant difference in radiation dose 

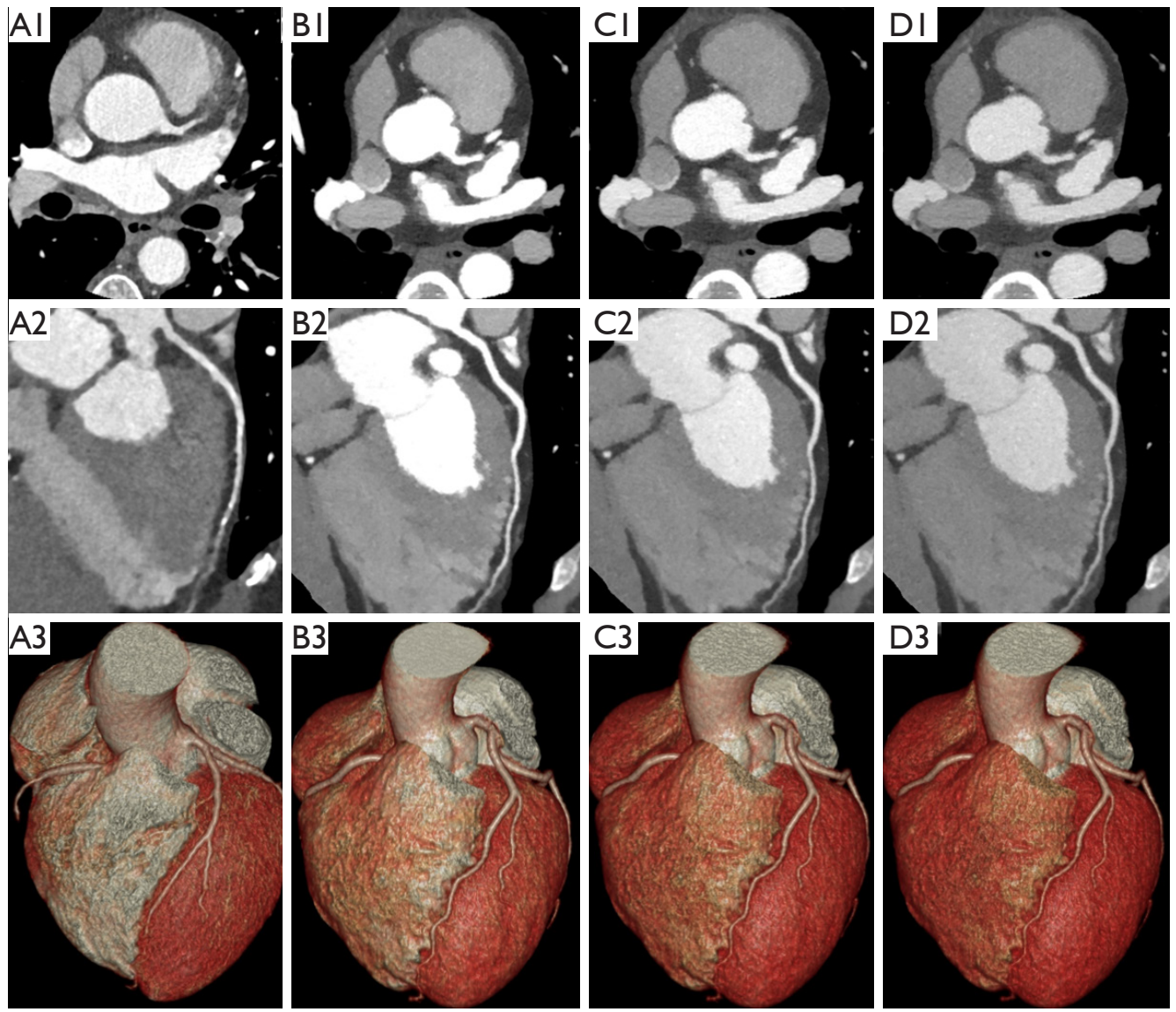

Figure 2 A 51-year-old woman with chest pain in Group A (body weight, $55 \mathrm{~kg}$; contrast media dose, $44 \mathrm{~mL}$; effective dose, $1.43 \mathrm{mSv}$ ) (A1A3) and a 46-year-old woman with chest pain in Group B-D (body weight, 60 kg; contrast media dose, $24 \mathrm{~mL}$; effective dose, $1.57 \mathrm{mSv}$ ) (B1B3; C1-C3; D1-D3). Transverse images, multi-planar reformation, and volume rendering images of the LAD were obtained in each group. In the A1, B1, C1, and D1 panels, the region of interest (in the aortic root at the origin of the left main coronary artery) showed a mean signal density of 316, 451, 363, and $307 \mathrm{HU}$, and a mean noise of 19.30, 22.53, 19.18, and 19.29. Image quality Likert scores in Group C were the best $(5 / 5 / 5 / 5)(\mathrm{C} 1-\mathrm{C} 3)$. LAD, left anterior descending.

between the routine iodine load group and the half iodine load group (DLP and ED): $132.66 \pm 38.36 \mathrm{mGy} \cdot \mathrm{cm}$ and $1.83 \pm 0.51 \mathrm{mSv}$ (routine iodine load), and $128.73 \pm 37.81$ $\mathrm{mGy} \cdot \mathrm{cm}$ and $1.80 \pm 0.53 \mathrm{mSv}$ (half iodine load) (both $\mathrm{P}>0.05)$. The average iodine loads for the routine iodine load and the half iodine load groups were $15.33 \pm 2.26 \mathrm{vs}$. $7.48 \pm 1.14 \mathrm{~g}$, respectively $(\mathrm{P}<0.001)$.

\section{Discussion}

We investigated the image quality of coronary CTA spectral reconstructions obtained from SDCT using reduced weight-based iodinated contrast and compared them with polychromatic images obtained using routine contrast protocol. We found that low VMI at $50 \mathrm{keV}$ provided better image interpretability and improved SNR and CNR with half iodine load, in comparison with the polychromatic reconstructions obtained using full iodine load.

VMI at low energies improves contrast enhancement and thus helps salvage suboptimal vascular studies or enable the use of a low dose of intravenous contrast (33). For patients who are clinically indicated for coronary CTA but have high renal insufficiency, a low-dose iodine regimen may be a viable option to reduce the incidence of CIN and provide a direct benefit to patients in terms of renal protection. Although VMI is widely used in various angiographic imaging in other DECT (pulmonary, aorta, abdominal, and lower-extremity CTA) $(17,20-24)$, it has only had limited 
Table 3 Comparison of objective observation indices of image quality among all groups

\begin{tabular}{|c|c|c|c|c|c|c|c|}
\hline Item & Group A (120 kVp) & Group B (45 keV) & Group C (50 keV) & Group D (55 keV) & $P$ value (A vs. B) & $P$ value (A vs. $C$ ) & $P$ value (A vs. $D)$ \\
\hline Aortic root & $317.28 \pm 37.79$ & $458.56 \pm 71.60$ & $374.86 \pm 57.64$ & $310.59 \pm 46.80$ & $<0.05$ & $<0.05$ & 0.84 \\
\hline Distal LAD & $267.96 \pm 43.33$ & $369.51 \pm 87.12$ & $307.10 \pm 65.58$ & $260.03 \pm 60.87$ & $<0.05$ & $<0.05$ & 0.40 \\
\hline Distal LCX & $272.57 \pm 45.87$ & $383.40 \pm 79.82$ & $321.46 \pm 54.17$ & $267.75 \pm 49.50$ & $<0.05$ & $<0.05$ & 0.56 \\
\hline \multicolumn{8}{|l|}{ SD } \\
\hline Aortic root & $19.31 \pm 2.76$ & $21.53 \pm 4.70$ & $19.94 \pm 4.16$ & $18.93 \pm 3.86$ & $<0.05$ & 0.75 & 0.96 \\
\hline \multicolumn{8}{|l|}{ SNR } \\
\hline Aortic root & $16.87 \pm 3.82$ & $21.93 \pm 4.24$ & $19.31 \pm 3.70$ & $16.85 \pm 3.26$ & $<0.05$ & $<0.05$ & 0.97 \\
\hline Distal RCA & $14.53 \pm 3.29$ & $18.92 \pm 5.32$ & $16.81 \pm 4.32$ & $14.78 \pm 3.98$ & $<0.05$ & $<0.05$ & 0.69 \\
\hline \multicolumn{8}{|l|}{ CNR } \\
\hline Aortic root & $21.91 \pm 4.61$ & $29.03 \pm 5.54$ & $26.14 \pm 4.93$ & $23.24 \pm 4.51$ & $<0.05$ & $<0.05$ & 0.06 \\
\hline Distal LAD & $19.24 \pm 4.01$ & $25.00 \pm 6.69$ & $22.84 \pm 5.76$ & $20.69 \pm 5.72$ & $<0.05$ & $<0.05$ & 0.07 \\
\hline Distal LCX & $19.46 \pm 3.95$ & $25.64 \pm 6.33$ & $23.64 \pm 5.83$ & $20.03 \pm 4.92$ & $<0.05$ & $<0.05$ & 0.39 \\
\hline Distal RCA & $19.58 \pm 4.00$ & $26.03 \pm 6.42$ & $23.64 \pm 5.50$ & $20.18 \pm 5.20$ & $<0.05$ & $<0.05$ & 0.35 \\
\hline
\end{tabular}

Data are presented as the mean \pm standard deviation or number. CT, computed tomography; SD, standard deviation; SNR, signal-to-noise ratio; CNR, contrast-to-noise ratio; LAD, left anterior descending artery; LCX, left circumflex artery; RCA, right coronary artery.

application in coronary CTA. Unlike source-based dualenergy approaches, the detector-based technology used here with a fast gantry rotation speed of $0.27 \mathrm{~s}$ enables simultaneous acquisition and generation of low- and highenergy photons that are aligned and registered in both spatial and temporal domains with little or no time lag, which is critical for coronary imaging (33). Additionally, the application of appropriate noise models and the near-perfect alignment of energy-sensitive projection data results in the cancellation of the anti-correlated noise which otherwise could result in higher noise (and thereby reduced SNR and CNR) and lower VMI levels, thereby offering a potential of further reductions of contrast volumes.

Results from our study show that 45 and $50 \mathrm{keV}$ VMI in coronary CTA can increase contrast in the lumen, and improve CNR in half iodine load compared to polychromatic images reconstructed from scans performed with routine iodine load. The photon energy of 45-55 keV is closer to the k-edge of iodine $(33 \mathrm{keV})$, increasing the photoelectric effect and leading to considerable or even better contrast and CNR with a half dose contrast media. Some investigators have reported that the optimal vascular attenuation in coronary CTA is about $350 \mathrm{HU}$ (34). Higher attenuation could increase calcium blooming and affect the lumen and the wall assessment, leading to an overestimation of stenosis. Consequently, in this study, we have also determined that $45 \mathrm{keV}$ may not be an optimum choice.

Noise is an important factor in ensuring diagnostic confidence in the assessment of coronary arteries. Previous studies have shown that image noise is inversely associated with the energy level; as monoenergetic $\mathrm{keV}$ decreases, the image noise increases $(35,36)$. In this study, we found that the image noise in VMI reconstructions at $50-55 \mathrm{keV}$ is basically the same as that in the polychromatic images. Compared with DECT studies using the rapid $\mathrm{kVp}$ switching technique, coronary CTA using SDCT has lower noise, and the noise value (18.9-19.9) in this study is lower than that of previous studies $(27.5 \pm 8.9,26.9 \pm 12.5)$ $(18,19)$. This can be attributed to the suppression of the anti-correlated noise using SDCT with the exactly matched 

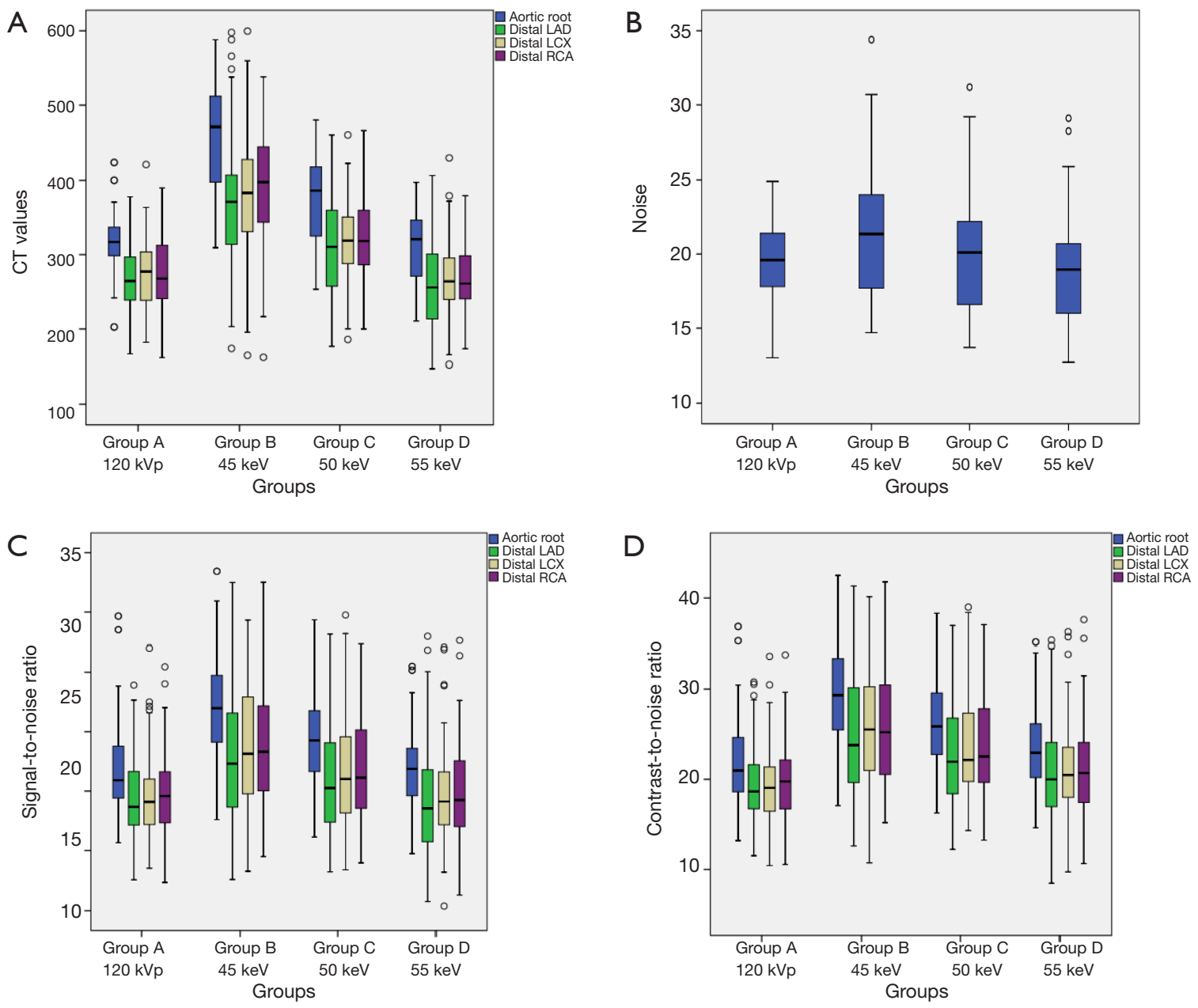

Figure 3 The boxplots of CT values (A), noise (B), signal-to-noise ratio (C), and contrast-to-noise ratio (D) in the aortic root, distal LAD, distal LCX, and distal RCA for the routine-dose Group A (120 kVp) and half-dose Group B-D (45/50/55 keV). CT, computed tomography; LAD, left anterior descending; LCX, left circumflex; RCA, right coronary artery.

high- and low-energy datasets generated without the need for angular and temporal interpolation (29).

In contrast, rapid $\mathrm{kVp}$ switching DECT VMI needs angular interpolation to reconstruct images within the projection domain. DECT approaches using dual source and twin beam only allow image reconstruction within the image space domain. Therefore, VMI from these systems can result in increased noise compared to conventional images obtained using comparable radiation doses (37). Low noise throughout the spectrum using SDCT may help us to take advantage of the full benefits of VMI, including improved contrast signal, improved lesion conspicuity and reduced artifacts $(38,39)$. Low noise can also result in increased SNR and CNR as shown in our study.
In this study, the increased contrast enhancement in the coronary lumen and noise reduction was obtained with a significantly reduced contrast media load in the half-dose group to $7.48 \pm 1.14 \mathrm{~g}$ compared to $15.33 \pm 2.26 \mathrm{~g}$ in the routine-dose group. At this dose, $50 \mathrm{keV}$ imaging has shown to achieve better image quality than routinedose polychromatic images. Previously, Raju et al. and Carrascosa et al. $(18,19)$ used rapid $\mathrm{kVp}$ switching DECT to obtain the best image quality at $60 \mathrm{keV}$. The contrast media load in their study was 11.2 and $12.5 \mathrm{~g}$, which was significantly higher than that of ours, and the SNR $(12.0 \pm 3.9$

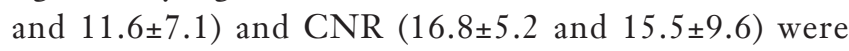
lower than that of our study $(19.3 \pm 3.7$ and $26.1 \pm 4.9)$. Since, spectral image reconstruction method of SDCT can provide 
an increased balance between the image signal and image noise at lower monoenergetic levels (40-60 keV), further improvement in signal and SNR are achieved.

The radiation dose in dual-energy coronary CTA has been a cause of concern, with some approaches imposing a higher radiation burden compared to a low $\mathrm{kVp}$ scanning technique (40). A comparison of patient radiation dose on different CT scanner models is highly dependent on the scan mode used and the numerous scan parameters selected (41). The radiation dose of SDCT coronary CTA in our study was $1.80 \pm 0.53 \mathrm{mSv}$, which was not statistically different from the routine polychromatic CT and was lower than the other source-based rapid $\mathrm{kVp}$ switching DECT approaches $(2.2-3.8 \mathrm{mSv})(18,19)$. Source-based DECT approaches that involved dual-source CT that typically uses the traditional helical retrospective scan protocols at a higher radiation dose (42). Lower effective radiation dose can be obtained via prospectively ECG-triggered highpitch spiral modes combined with lower polychromatic tube energies but in the conventional mode with no dual energy information available $(43,44)$. DECT investigations for coronary imaging using this mode of scanning are, therefore, currently lacking. Several studies have shown that SDCT can obtain dual energy information in a wide range of clinical indications at $120 \mathrm{kVp}$ tube potential, and the dose level is similar to or significantly lower than that of the traditional conventional CT $(39,45)$. Likewise, our findings suggest that this can be extended to coronary CTA as well, similar to the work of Yi et al. (29).

While the SDCT scanner used in this study had a limited z-coverage, the presence of spatial and temporal alignment in the projection domain without any sacrifice to temporal resolution makes it very useful for imaging small moving structures (such as coronary arteries). Thus it does not encounter some of the limitations of other source-based dual-energy scanners (such as limited field-of-view, rotation time, sacrificing temporal resolution, etc.) while at the same time offering workflow benefits without the need to select special dual-energy scan modes ahead of time $(33,41,46,47)$.

This study had some limitations. Firstly, we did not study the diagnostic accuracy and performance of monoenergetic images compared to invasive coronary angiography (ICA) or routine-dose polychromatic images for detecting coronary stenosis. Our assessment instead focused on quantitative and qualitative measurements of image quality. Secondly, only a single spectral reconstruction level (Spectral Level 4) was used. Further research is needed on the use of different spectral reconstruction levels. Thirdly, we did not test our protocol in high-risk patients, nor did we assess post-scan serum creatinine levels. Therefore, we cannot comment on the incidence of CIN in any of our groups, nor can we comment on the safety or ability to reduce the incidence of CIN in high-risk populations. Finally, image quality comparisons with different scanner models were not involved in our study.

\section{Conclusions}

In summary, $50 \mathrm{keV}$ monoenergetic images from prospectively ECG-gated coronary CTA performed on a dual-layer SDCT can provide equivalent or improved coronary image quality with half contrast media load compared to polychromatic coronary CTA with routine contrast load.

\section{Acknowledgments}

The authors would like to acknowledge Mani Vembar and Hui Yao for their assistance with CT technology.

Funding: This study was supported by the National Natural Science Foundation of China (Grant No. 81301221), Innovative Research Team of Liaoning Educational Committee (No. LT2014017), and 345 Talent Project in Shengjing Hospital of China Medical University. The funders had no role in study design, data collection and analysis, decision to publish, or preparation of the manuscript.

\section{Footnote}

Conflicts of Interest: The authors have no conflicts of interest to declare.

Ethical Statement: This study was approved by the ethics committee of Shengjing Hospital of China Medical University, and written informed consent was obtained from all patients.

Open Access Statement: This is an Open Access article distributed in accordance with the Creative Commons Attribution-NonCommercial-NoDerivs 4.0 International License (CC BY-NC-ND 4.0), which permits the noncommercial replication and distribution of the article with the strict proviso that no changes or edits are made and the original work is properly cited (including links to both the formal publication through the relevant DOI and the license). 
See: https://creativecommons.org/licenses/by-nc-nd/4.0/.

\section{References}

1. Kerl JM, Schoepf UJ, Zwerner PL, Bauer RW, Abro JA, Thilo C, Vogl TJ, Herzog C. Accuracy of coronary artery stenosis detection with CT versus conventional coronary angiography compared with composite findings from both tests as an enhanced reference standard. Eur Radiol 2011;21:1895-903.

2. Janne d'Othée B, Siebert U, Cury R, Jadvar H, Dunn EJ, Hoffmann U. A systematic review on diagnostic accuracy of CT-based detection of significant coronary artery disease. Eur J Radiol 2008;65:449-61.

3. Marwan M, Pflederer T, Schepis T, Seltmann M, Klinghammer L, Muschiol G, Ropers D, Daniel WG, Achenbach S. Accuracy of dual-source CT to identify significant coronary artery disease in patients with uncontrolled hypertension presenting with chest pain: comparison with coronary angiography. Int J Cardiovasc Imaging 2012;28:1173-80.

4. Hou Y, Zheng J, Wang Y, Yu M, Vembar M, Guo Q. Optimizing radiation dose levels in prospectively electrocardiogram-triggered coronary computed tomography angiography using iterative reconstruction techniques: a phantom and patient study. PLoS One 2013;8:e56295.

5. Pan YN, Li AJ, Chen XM, Wang J, Ren DW, Huang QL. Coronary computed tomographic angiography at low concentration of contrast agent and low tube voltage in patients with obesity: a feasibility study. Acad Radiol 2016;23:438-45.

6. From AM, Al Badarin FJ, McDonald FS, Bartholmai BJ, Cha SS, Rihal CS. Iodixanol versus low-osmolar contrast media for prevention of contrast induced nephropathy: meta-analysis of randomized, controlled trials. Circ Cardiovasc Interv 2010;3:351-8.

7. McDonald JS, McDonald RJ, Carter RE, Katzberg RW, Kallmes DF, Williamson EE. Risk of intravenous contrast material-mediated acute kidney injury: a propensity scorematched study stratified by baseline-estimated glomerular filtration rate. Radiology 2014;271:65-73.

8. McDonald JS, McDonald RJ, Comin J, Williamson EE, Katzberg RW, Murad MH, Kallmes DF. Frequency of acute kidney injury following intravenous contrast medium administration: a systematic review and meta-analysis. Radiology 2013;267:119-28.

9. Heinrich MC, Kuhlmann MK, Grgic A, Heckmann M,
Kramann B, Uder M. Cytotoxic effects of ionic highosmolar, nonionic monomeric, and nonionic iso-osmolar dimeric iodinated contrast media on renal tubular cells in vitro. Radiology 2005;235:843-9.

10. Davenport MS, Cohan RH, Khalatbari S, Ellis JH. The challenges in assessing contrast-induced nephropathy: where are we now? AJR Am J Roentgenol 2014;202:784-9.

11. Nyman U, Björk J, Aspelin P, Marenzi G. Contrast medium dose-to-GFR ratio: a measure of systemic exposure to predict contrast-induced nephropathy after percutaneous coronary intervention. Acta Radiol 2008;49:658-67.

12. Oda S, Takaoka H, Katahira K, Honda K, Nakaura T, Nagayama Y, Taguchi N, Kidoh M, Utsunomiya D, Funama Y, Noda K, Oshima S, Yamashita Y. Low contrast material dose coronary computed tomographic angiography using a dual-layer spectral detector system in patients at risk for contrast-induced nephropathy. $\mathrm{Br} \mathrm{J}$ Radiol 2019;92:20180215.

13. Kok M, Mihl C, Hendriks BM, Altintas S, Kietselaer BL, Wildberger JE, Das M. Optimizing contrast media application in coronary CT angiography at lower tube voltage: evaluation in a circulation phantom and sixty patients. Eur J Radiol 2016;85:1068-74.

14. Iyama Y, Nakaura T, Yokoyama K, Kidoh M, Harada K, Oda S, Tokuyasu S, Yamashita Y. Low-contrast and lowradiation dose protocol in cardiac computed tomography: usefulness of low tube voltage and knowledge-based iterative model reconstruction algorithm. J Comput Assist Tomogr 2016;40:941-7.

15. Wu Q, Wang Y, Kai H, Wang T, Tang X, Wang X, Pan C. Application of $80-\mathrm{kVp}$ tube voltage, low-concentration contrast agent and iterative reconstruction in coronary CT angiography: evaluation of image quality and radiation dose. Int J Clin Pract 2016;70 Suppl 9B:B50-5.

16. De Santis D, Eid M, De Cecco CN, Jacobs BE, Albrecht MH, Varga-Szemes A, Tesche C, Caruso D, Laghi A, Schoepf UJ. Dual-energy computed tomography in cardiothoracic vascular imaging. Radiol. Clin. North Am 2018;56:521-34.

17. Almutairi A, Sun Z, Poovathumkadavi A, Assar T. Dual energy CT angiography of peripheral arterial disease: feasibility of using lower contrast medium volume. PLoS One 2015;10:e139275.

18. Raju R, Thompson AG, Lee K, Precious B, Yang TH, Berger A, Taylor C, Heilbron B, Nguyen G, Earls J, Min J, Carrascosa P, Murphy D, Hague C, Leipsic JA. Reduced iodine load with CT coronary angiography using dual- 
energy imaging: a prospective randomized trial compared with standard coronary CT angiography. J Cardiovasc Comput Tomogr 2014;8:282-8.

19. Carrascosa P, Leipsic JA, Capunay C, Deviggiano A, Vallejos J, Goldsmit A, Rodriguez-Granillo GA. Monochromatic image reconstruction by dual energy imaging allows half iodine load computed tomography coronary angiography. Eur J Radiol 2015;84:1915-20.

20. Lee JW, Lee G, Lee NK, Moon JI, Ju YH, Suh YJ, Jeong YJ. Effectiveness of adaptive statistical iterative reconstruction for 64-Slice dual-energy computed tomography pulmonary angiography in patients with a reduced iodine load: comparison with standard computed tomography pulmonary angiography. J Comput Assist Tomogr 2016;40:777-83.

21. Delesalle MA, Pontana F, Duhamel A, Faivre JB, Flohr T, Tacelli N, Remy J, Remy-Jardin M. Spectral optimization of chest CT angiography with reduced iodine load: experience in 80 patients evaluated with dual-source, dualenergy CT. Radiology 2013;267:256-66.

22. Shuman WP, Chan KT, Busey JM, Mitsumori LM, Koprowicz KM. Dual-energy CT Aortography with 50\% Reduced Iodine Dose Versus Single-energy CT Aortography with Standard Iodine Dose. Acad Radiol 2016;23:611-8.

23. Shuman WP, O'Malley RB, Busey JM, Ramos MM, Koprowicz KM. Prospective comparison of dual-energy CT aortography using 70\% reduced iodine dose versus single-energy CT aortography using standard iodine dose in the same patient. Abdom Radiol (NY) 2017;42:759-65.

24. Agrawal MD, Oliveira GR, Kalva SP, Pinho DF, Arellano RS, Sahani DV. Prospective comparison of reducediodine-dose virtual monochromatic imaging dataset from dual-energy CT angiography with standard-iodine-dose single-energy CT angiography for abdominal aortic aneurysm. AJR Am J Roentgenol 2016;207:W125-W132.

25. Hickethier T, Baeßler B, Kroeger JR, Doerner J, Pahn G, Maintz D, Michels G, Bunck AC. Monoenergetic reconstructions for imaging of coronary artery stents using spectral detector CT: In-vitro experience and comparison to conventional images. J Cardiovasc Comput Tomogr 2017;11:33-9.

26. Rassouli N, Chalian H, Rajiah P, Dhanantwari A, Landeras L. Assessment of 70-keV virtual monoenergetic spectral images in abdominal CT imaging: A comparison study to conventional polychromatic $120-\mathrm{kV}$ images. Abdom Radiol (NY) 2017;42:2579-86.

27. Rassouli N, Etesami M, Dhanantwari A, Rajiah P.
Detector-based spectral CT with a novel dual-layer technology: principles and applications. Insights Imaging 2017;8:589-98.

28. van Hamersvelt RW, Eijsvoogel NG, Mihl C, de Jong PA, Schilham AMR, Buls N, Das M, Leiner T, Willemink MJ. Contrast agent concentration optimization in CTA using low tube voltage and dual-energy CT in multiple vendors: a phantom study. Int J Cardiovasc Imaging 2018;34:1265-75.

29. Yi Y, Zhao XM, Wu RZ, Wang Y, Vembar M, Jin ZY, Wang YN. Low dose and low contrast medium coronary CT angiography using dual-layer spectral detector CT. Int Heart J 2019;60:608-17.

30. Abbara S, Blanke P, Maroules CD, Cheezum M, Choi AD, Han BK, Marwan M, Naoum C, Norgaard BL, Rubinshtein R, Schoenhagen P, Villines T, Leipsic J. SCCT guidelines for the performance and acquisition of coronary computed tomographic angiography: a report of the society of Cardiovascular Computed Tomography Guidelines Committee: endorsed by the North American Society for Cardiovascular Imaging (NASCI). J Cardiovasc Comput Tomogr 2016;10:435-49.

31. Zhang C, Zhang Z, Yan Z, Xu L, Yu W, Wang R. 320-row CT coronary angiography: effect of $100-\mathrm{kV}$ tube voltages on image quality, contrast volume, and radiation dose. Int J Cardiovasc Imaging 2011;27:1059-68.

32. Hou Y, Ma Y, Fan W, Wang Y, Yu M, Vembar M, Guo Q. Diagnostic accuracy of low-dose 256-slice multi-detector coronary CT angiography using iterative reconstruction in patients with suspected coronary artery disease. Eur Radiol 2014;24:3-11.

33. Rajiah P, Abbara S, Halliburton SS. Spectral detector CT for cardiovascular applications. Diagn Interv Radiol 2017;23:187-93.

34. Fei X, Du X, Yang Q, Shen Y, Li P, Liao J, Li K. 64MDCT coronary angiography: phantom study of effects of vascular attenuation on detection of coronary stenosis. AJR Am J Roentgenol 2008;191:43-9.

35. Apfaltrer P, Sudarski S, Schneider D, Nance JW, Haubenreisser H, Fink C, Schoenberg SO, Henzler T. Value of monoenergetic low-kV dual energy CT datasets for improved image quality of CT pulmonary angiography. Eur J Radiol 2014;83:322-8.

36. Bellini D, Gupta S, Ramirez-Giraldo JC, Fu W, Stinnett SS, Patel B, Mileto A, Marin D. Use of a Noise optimized monoenergetic algorithm for patientsize independent selection of an optimal energy level during dual-energy CT of the pancreas. J Comput Assist Tomogr 2017;41:39-47. 
37. Große Hokamp N, Gilkeson R, Jordan MK, Laukamp KR, Neuhaus VF, Haneder S, Halliburton SS, Gupta A. Virtual monoenergetic images from spectral detector CT as a surrogate for conventional CT images: unaltered attenuation characteristics with reduced image noise. Eur J Radiol 2019;117:49-55.

38. Kalisz K, Rassouli N, Dhanantwari A, Jordan D, Rajiah P. Noise characteristics of virtual monoenergetic images from a novel detector-based spectral CT scanner. Eur J Radiol 2018;98:118-25.

39. Doerner J, Hauger M, Hickethier T, Byrtus J, Wybranski C, Große Hokamp N, Maintz D, Haneder S. Image quality evaluation of dual-layer spectral detector CT of the chest and comparison with conventional CT imaging. Eur J Radiol 2017;93:52-8.

40. Danad I, Fayad ZA, Willemink MJ, Min JK. New applications of cardiac computed tomography: dual-energy, spectral, and molecular CT Imaging. JACC Cardiovasc Imaging 2015;8:710-23.

41. Lewis MA, Pascoal A, Keevil SF, Lewis CA. Selecting a CT scanner for cardiac imaging: the heart of the matter. Br J Radiol 2016;89:20160376.

42. Symons R, Choi Y, Cork TE, Ahlman MA, Mallek M, Bluemke DA, Sandfort V. Optimized energy of spectral coronary CT angiography for coronary plaque detection and quantification. J Cardiovasc Comput Tomogr 2018;12:108-14.

43. Hell MM, Bittner D, Schuhbaeck A, Muschiol G, Brand M,
Lell M, Uder M, Achenbach S, Marwan M. Prospectively ECG-triggered high-pitch coronary angiography with third-generation dual-source CT at $70 \mathrm{kVp}$ tube voltage: feasibility, image quality, radiation dose, and effect of iterative reconstruction. J Cardiovasc Comput Tomogr 2014;8:418-25.

44. Smettei OA, Sayed S, M Al Habib A, Alharbi F, Abazid RM. Ultra-fast, low dose high-pitch (FLASH) versus prospectively-gated coronary computed tomography angiography: Comparison of image quality and patient radiation exposure. J Saudi Heart Assoc 2018;30:165-71.

45. Hojjati M, Van Hedent S, Rassouli N, Tatsuoka C, Jordan D, Dhanantwari A, Rajiah P. Quality of routine diagnostic abdominal images generated from a novel detector-based spectral CT scanner: a technical report on a phantom and clinical study. Abdom Radiol (NY) 2017;42:2752-9.

46. Lu X, Lu Z, Yin J, Gao Y, Chen X, Guo Q. Effects of radiation dose levels and spectral iterative reconstruction levels on the accuracy of iodine quantification and virtual monochromatic CT numbers in dual-layer spectral detector CT: an iodine phantom study. Quant Imaging Med Surg 2019;9:188-200.

47. Li L, Zhao Y, Luo D, Yang L, Hu L, Zhao X, Wang Y, Liu W. Diagnostic value of single-source dual-energy spectral computed tomography in differentiating parotid gland tumors: initial results. Quant Imaging Med Surg 2018;8:588-96.
Cite this article as: Huang X, Gao S, Ma Y, Lu X, Jia Z, Hou Y. The optimal monoenergetic spectral image level of coronary computed tomography (CT) angiography on a dual-layer spectral detector CT with half-dose contrast media. Quant Imaging Med Surg 2020;10(3):592-603. doi: 10.21037/ qims.2020.02.17 